\title{
Functional and radiographic outcomes of hallux valgus correction by mini-invasive surgery with Reverdin-Isham and Akin percutaneous osteotomies: a longitudinal prospective study with a 48-month follow-up
}

Carlo Biz $^{1 *}$, Michele Fosser ${ }^{1}$, Miki Dalmau-Pastor ${ }^{2,3}$, Marco Corradin ${ }^{1}$, Maria Grazia Rodà ${ }^{4}$, Roberto Aldegheri ${ }^{1}$ and Pietro Ruggieri ${ }^{1}$

\begin{abstract}
Background: Minimally invasive surgery (MIS) represents one of the most innovative surgical treatments of hallux valgus (HV). However, long-term outcomes still remain a matter of discussion within the orthopaedic community. The purpose of this longitudinal prospective study was to evaluate radiographic and functional outcomes in patients with mild-to-severe HV who underwent Reverdin-Isham and Akin percutaneous osteotomy, following exostosectomy and lateral release.
\end{abstract}

Methods: Eighty patients with mild-to-severe symptomatic HV were treated by MIS. Clinical evaluation was assessed preoperatively, as well as at 3 and 12 months after surgery and at final follow-up of 48 months, using the American Orthopaedic Foot and Ankle Society (AOFAS) hallux grading system. Patient satisfaction and complications were recorded. Computer-assisted measurement of antero-posterior radiographs was taken preoperatively, as well as at 3 and 12 months after surgery and at 48-month follow-up, analysing the intermetatarsal angle (IMA), the hallux valgus angle (HVA), the distal metatarsal articular angle (DMAA) and the tibial sesamoid position. Also, the bridging bone/callus formation was evaluated at the different radiographic follow-ups, while the articular surface congruency and the metatarsal index were calculated only preoperatively and at the last follow-up. Patient satisfaction was assessed using the visual analogue score (VAS). Statistical analysis was carried out using the paired $t$ test. Statistical significance was set at $p<0.05$.

Results: The mean AOFAS score was 87.15 points at the final follow-up of 48 months, and the VAS score was 8.35/10. The post-operative radiographic assessments showed a statistically significant improvement compared with preoperative values. The mean corrections of each angular value at the last follow-up were as follows: IMA $3.90^{\circ}, \mathrm{HVA} 12.50^{\circ}$, DMAA $4.72^{\circ}$ and a tibial sesamoid position of 1.10. The articular surface was congruent in $77(96.25 \%)$ cases and incongruent only in 3 (3.75\%). The complete healing of the osteotomies was achieved in all series at 3-month follow-up. However, the results obtained in the correction of the severe HV deformities were less encouraging. (Continued on next page)

\footnotetext{
* Correspondence: carlo.biz@unipd.it

'Orthopaedic Clinic, Department of Surgery, Oncology and Gastroenterology

DisCOG, University of Padua, via Giustiniani 2, 35128 Padova, Italy

Full list of author information is available at the end of the article
} 
(Continued from previous page)

Conclusions: Minimally invasive surgery with Reverdin-Isham and Akin percutaneous osteotomy, in combination with previous exostosectomy and subsequent lateral soft-tissue release, is a safe, effective and reliable procedure for correction of mild-to-moderate HV. However, it requires a long learning curve because of the inherent difficulty of the mixed different surgical procedures.

Trial registration: ClinicalTrials.gov PRS Protocol Registration and Results System: NCT02886221

Keywords: Hallux valgus, Reverdin-Isham osteotomy, Akin osteotomy, Minimally invasive surgery, Percutaneous distal osteotomy, First ray, Forefoot

\section{Background}

Hallux valgus (HV) is a common, complex and progressive deformity of the forefoot with multiple clinical symptoms and a multifactorial aetiology [1]. Painful HV is more frequent in women between 40 and 60 years old, although it can appear in younger people because of biomechanical influence, hind and midfoot pathologies or sports activities, which might cause an overload on the first ray $[2,3]$. For its correction, a wide variety of bony procedures are described, associated or not with soft tissue release, including osteotomies at the level of the head, midshaft and base of the first metatarsal, as well as arthrodesis of the first metatarso-cuneiform joint [4-7]. This demonstrates the complexity of the disease and the lack of a unique and most appropriate treatment, the choice of which continues to generate controversy [8].

At present, minimally invasive surgery (MIS) performed with minimal skin incisions (1-3 mm), an intraoperative image intensificator and without internal fixation [9] represents one of the most innovative approaches in forefoot surgery. This percutaneous dynamic management combines different procedures, most arising from the traditional open distal metatarsal osteotomy, in a mixed surgical strategy, according to the complexity of the deformity to be corrected [10-14]. These methods are rapidly becoming popular, as they are quick to perform, allow 1-day hospitalization, decrease post-operative morbidity as well as recovery and rehabilitation times, and chiefly because they are better accepted by patients $[9,15]$.

Although the most commonly performed percutaneous procedures have already been well described, providing equal effectiveness, sometimes superior, to traditional open surgery [16], their use is not equally accepted and their outcomes still remain a matter of discussion in the orthopaedic community, particularly in cases where no internal fixation is used $[17,18]$. The Reverdin-Isham percutaneous osteotomy was described as a novel intra-articular medial closing wedge osteotomy of the distal metatarsal, in combination with an Akin osteotomy, both performed without fixation, to align the first ray by medial rotation of the first metatarsal head and distal metatarsal articular angle (DMAA) correction [15, 19-26]. Reverdin-Isham is not a complete osteotomy, as the MTT-1 lateral cortex is preserved; the closing wedge ensures contact of the metatarsal head with the metaphysis, and a special bandage is applied after surgery. In this way, no internal fixation is necessary. This allows the osteotomy to heal with the toe in its proper position, due to early weight bearing.

Since the end of the last century, MIS became widespread first in Spain and then in Europe by M. De Prado and P.L. Ripoll through their surgical practices and international theoretical-cadaveric courses, supported by the anatomical studies of Pau Golanó [20]. In 2002, the group GRECMIP (Groupe de Recherche et d'Enseignement en Chirurgie Mini-Invasive du Pied) began a project to develop and promote this new surgical treatment [26]. However, to the best of our knowledge, no previous study has evaluated the long-term results of this technique. Thus, the aim of this prospective study was to evaluate the radiographic and clinical outcomes of patients with mild-to-severe HV treated by MIS with Reverdin-Isham and Akin percutaneous osteotomy, following exostosectomy and lateral soft-tissue release.

\section{Methods}

Between May 2010 and May 2012, a consecutive series of 80 Caucasian patients with diagnosis of mild-tosevere HV were enrolled in this prospective study at our institution and underwent the Reverdin-Isham percutaneous osteotomy, following percutaneous Akin osteotomy and percutaneous lateral soft-tissue release. All of these operative procedures were performed by a single surgeon, the senior author (C.B.), who followed and checked the patients personally during the postoperative period. All subjects participating in this prospective study received a thorough explanation of the risks and benefits of inclusion and gave their oral and written informed consent to publish the data. Approval from the General Clinical Directorate of our institution was obtained to introduce the novel technique before starting the operations. The study was performed in accordance with the ethical standards of the 1964 Declaration of Helsinki as revised in 2000 and those of Good Clinical Practice. 


\section{Inclusion and exclusion criteria}

Patients with diagnosis of mild to severe HV were enrolled consecutively and prospectively with precise inclusion criteria over a 2-year period. Ages ranged from 25 to 80 years. Only symptomatic patients with severe pain were included in this study. Exclusion criteria were as follows: congenital deformities of the foot, hallux rigidus, previous first ray trauma or foot and ankle surgery, diagnosis of rheumatic, dismetabolic, neurologic, infective or psychiatric pathologies. Furthermore, patients were excluded if they had painful fixed lesser toe deformities, signs of metatarsalgia or Morton's neuroma.

\section{Surgical procedures}

The different procedures for MIS HV correction, as adopted by our institution, were performed as described by De Prado [20]. Among these specific tools, various burrs of different size and form, adapted for Mm960 (produced by Medic Micro, Switzerland), a modular power driver for MIS, were used. During the operation, the patient was in a supine position, with the operated foot protruding from the table. No ankle joint tourniquet was applied, as it is not required for this technique. Prophylactic antibiotic (Cefazolin $2 \mathrm{~g}$ ) was administered before surgery, and thromboembolic prophylaxis with Nadroparin Calcium injections was prescribed the same evening and for a 30-day period. Anaesthesia consisted in a conscious sedation in association with a regional ankle block, which combines five nerves: three superficial: saphenous, sural and superficial peroneal nerves, and two deep: tibial and deep peroneal nerves.

\section{Exostosectomy}

An incision of 3-5 mm long was made at the plantar side of the medial border of the first metatarsal head (Fig. 1a).
Through this medial approach, a small scalpel was introduced within the joint capsule of the metatarsophalangeal joint of the big toe. By a sweeping movement, the medial capsule was separated from the exostosis, subsequently using also a rasp (Fig. 1b). The location of this incision prevents damage of the dorsomedial cutaneous nerve of the hallux [20] (Fig. 1c). Then, a cylindrical burr $(3.1 \times 15 \mathrm{~mm})$ was introduced, and the dorsal medial prominence was removed from the first metatarsal head until a flat surface was obtained, assessed under manual palpation and fluoroscopic control. Finally, the bony detritus was extruded manually.

\section{Reverdin-Isham osteotomy}

Through the same incision used for the exostosectomy, a Shannon Isham burr $(2 \times 12 \mathrm{~mm})$ was introduced at the junction of metaphysis and epiphysis. It was applied to the flat bone surface, achieved previously by exostosectomy, at an angle of approximately $45^{\circ}$ to the long axis of the first metatarsal bone, keeping the articular cartilage surface of the first metatarsal head as reference point on the dorsal cortex, and the medial sesamoid bone as the reference point on the plantar cortex (Fig. 2a). In this position, under fluoroscopic control, the osteotomy was started following a distal-dorsal and proximal-plantar direction, extending until the lateral cortex, but without cutting it. At this point, the burr was slightly withdrawn in order to preserve a few millimetres of the lateral cortex, and the osteotomy of the plantar cortex was performed completely. Then, a Wedge burr $(3.1 \times 13 \mathrm{~mm}$ or $4.1 \times 13 \mathrm{~mm}$, depending on the DMAA value) was used to create a wedge with a medially oriented base. At the point of closing the wedge, osteoclasis of the preserved lateral cortex was achieved, modifying the

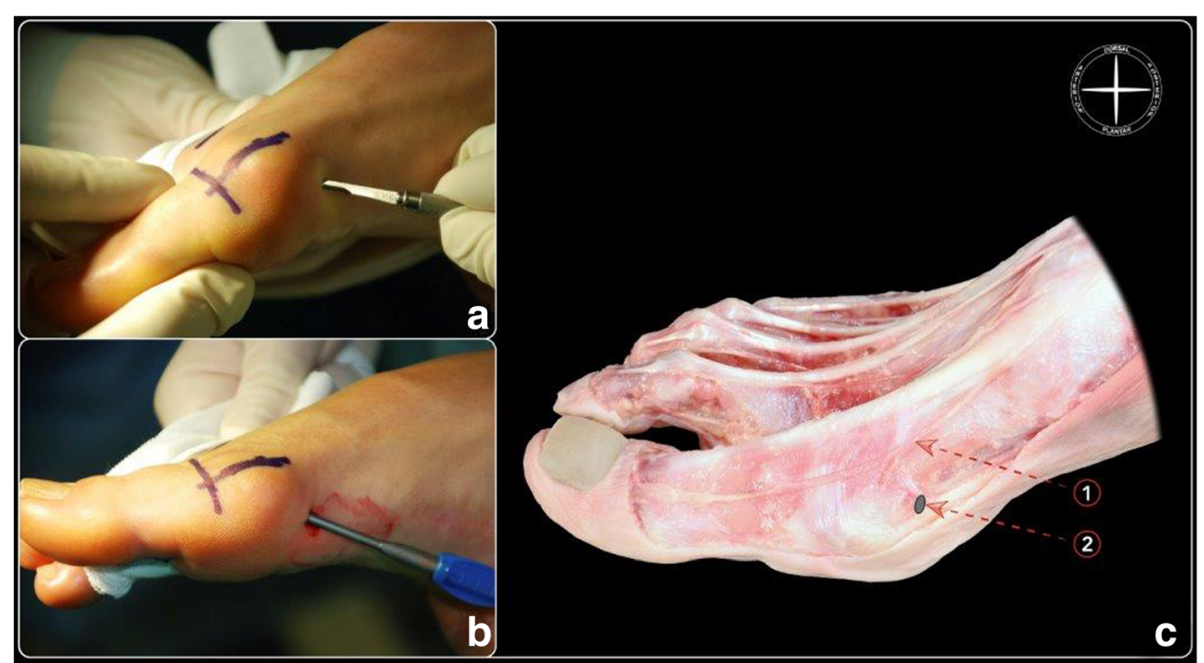

Fig. 1 Portal placement (a) and rasp introduction (b). The protocolised incision protects the dorsomedial cutaneous nerve of the hallux (c): 1 dorsomedial cutaneous nerve of the hallux, 2 point of incision for Reverdin-Isham osteotomy 


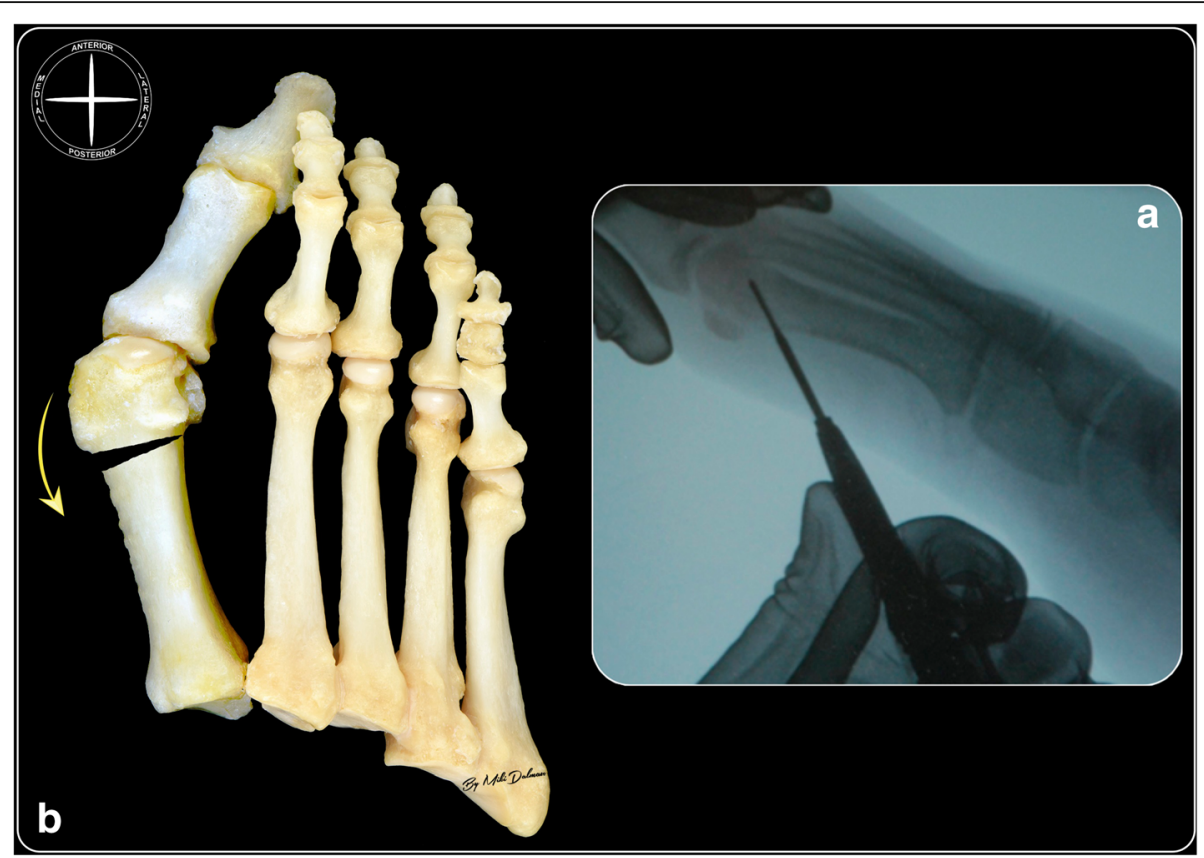

Fig. 2 Reverdin-Isham osteotomy: intraoperative fluoroscopic imagine shoving the proper position and inclination of the burr to respect the distal first metatarsal bone (a). The final result of an ideal closing wedge osteotomy with a medial base that corrects also the DMAA (b)

orientation of the articular surface, normalizing the DMAA value and adding intrinsic stability to the osteotomy by producing contact of the trabecular bone (Fig. 2b).

\section{Tenotomy of the adductor hallucis tendon and lateral capsulotomy}

A longitudinal skin incision was performed on the first web space, 2-3 mm lateral to the extensor hallucis longus tendon. The blade was longitudinally introduced in contact with the lateral surface of the base of the proximal phalanx; then, the blade was rotated $90^{\circ}$ laterally and the first toe forced in varus, causing the adductor hallucis tendon to be sectioned and the lateral part of the capsule joint to be cut. Movement of the blade was carefully controlled in order to avoid a complete capsulotomy, which could produce joint instability.

\section{Akin osteotomy}

Once lateral soft-tissue release was performed, a new incision 3 to $5 \mathrm{~mm}$ long on the lateral surface of the base of the proximal phalanx of the first toe was performed, just medial to the extensor tendons. Using a small scraper, the periosteum was removed from the lateral surface of the base of the proximal phalanx. Then, using a Wedge burr $(3.1 \times 13 \mathrm{~mm})$, a wedge osteotomy (with medial base) was performed; as in the osteotomy on the head of the first metatarsal, the lateral cortex was preserved. Closing of the osteotomy and osteoclasis of the lateral cortex was achieved by means of a forced varus movement of the toe.

\section{Bandage}

After completing the surgery with suture of the capsule and cutaneous sutures of related cuts, a bandage was applied. Because there is no osteosynthesis material in this surgery, the bandage is a very important tool in order to maintain the correction obtained with the operation. Consequently, its application was performed with the utmost care and attention. The first toe was gently placed in overcorrection. Then, with a tape for bandages, the bend of the crisscross bandage was traced between the first and second toes, crossing them over the medial aspect of the exostosectomy in order to reinforce the strength of the bandage. Gentle traction was used to maintain the toe in light hypercorrection and plantar inclination. Finally, the forefoot was covered with tubular gauzes, except for the distal part of the toes and nails (Fig. 3a).

\section{Post-operative protocol}

All patients followed the same post-operative protocol and were followed in the same standardized manner by the senior author (C.B.). The patients were allowed to walk as much as they could tolerate the same evening after surgery at discharge, using a rigid flat-soled orthopaedic shoe for the following 30-day period (Fig. 3b). Antero-posterior and lateral X-rays of nonweight-bearing feet were taken before the patients were discharged. We recommended a thromboembolic prophylaxis (Natrium Enoxaparin: 4000 IU/day) and an anti-edemigen therapy (Leucoselect, Lymphaselect and 


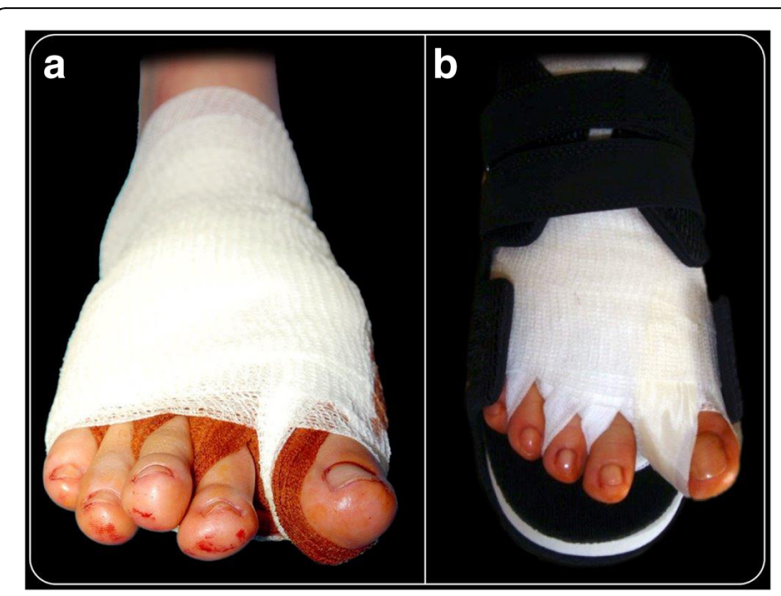

Fig. 3 Example of post-operative bandage (a) and rigid flat-soled orthopaedic shoe (b)

Bromelina: $1 \mathrm{cp} /$ day) for 30 days, starting from the day of the surgery. Moreover, an analgesic therapy was prescribed for 2 weeks with Etoricoxib (90 mg, $1 \mathrm{cp} /$ day) in the morning, also to prevent articular ossification; if pain persisted, Paracetamol/phosphate Codeine ( $1 \mathrm{~g}$, $\max \times 3 /$ day) was prescribed. All of the patients were seen once a week for a month in our out-patient clinic. The first visit was 8 days after surgery. The original bandage was removed and substituted by a simpler bandage, but always with a slight overcorrection. During the three weekly visits, the bandage was changed in the same way. One month after surgical treatment, the bandage was totally removed, and after taking anteroposterior weight bearing and lateral X-rays (and sesamoid view when possible), an interdigital silicone orthoses space maintainer was positioned between the first and second toes. Patients were instructed to wear it for 3 months to help the first toe maintain its correct position until complete osteotomy consolidation. They were then able to walk with comfortable shoes, allowing total load on the operated foot. The only recommendations for the patient were to be careful with rough surfaces, sports and any other activities with forefoot overload. No specific physiokinesis therapy was suggested to restart daily activities.

\section{Patient assessment}

The clinical and radiological analyses were carried out, respectively, by two independent investigators, the junior authors (M.F. and M.G.R.), not involved in the primary surgical treatment of the patients. The first is a resident of our clinic; the second is an orthopaedic surgeon of a different unit. For this study, all of the patients underwent clinical and radiographic assessment with the same protocol before surgery, as well as at 3 and 12 months and finally at 48 months after surgery, according to the
American Orthopaedic Foot and Ankle Society (AOFAS) accepted guidelines [27]. For methodological reasons, the immediate post-operative $\mathrm{X}$-rays at discharge, as well as the 1-month radiographic control, were not included for the radiographic evaluation: first, because it was a non-weight-bearing radiograph and, second, because, although it was weight bearing, in some cases, the sesamoid projection was not performed as the patients had pain or were afraid to excessively dorsiflex the big toe.

\section{Functional outcome measures}

The clinical preoperative evaluation included a complete clinical history of the patients, their main characteristics (gender, age at the time of surgery, affected side) and physical examination of the foot. The 100-point AOFAS hallux-metatarso-phalangeal-interphalangeal scale [28] was used to assess clinical outcomes, and the difference $(\Delta)$ between preoperative and post-operative median values was calculated. Furthermore, all patients were investigated with the visual analogue scale (VAS). Additionally, any complications were recorded.

\section{Radiographic outcome measures}

Routine standing antero-posterior, lateral and sesamoid $\mathrm{X}$-ray views were obtained before surgery and at different follow-ups, according to our protocol. They were analysed at our institution in a standardised manner using electronically computer-assisted measurements for weight-bearing radiographs. The following parameters were evaluated: intermetatarsal angle (IMA: normal value $<10^{\circ}$ ), proximal articular surface angle (DMAA: normal value $<6^{\circ}$ ), hallux valgus angle (HVA: normal value $<15^{\circ}$ ), tibial sesamoid position (using the recommended classification system by the American Foot and Ankle Society [29]), articular surface congruency, metatarsal index [30-32], callus formation in anteroposterior and lateral view radiographs and absence of radiolucent lines to determine bone union.

The relationship among the IMA, HVA values and tibial sesamoid displacement was then used to classify the deformities into three groups according to the presence of one of these Mann and Coughlin parameters $[1,28,29,33,34]$ :

a) Mild HV was defined as an IMA $\leq 11^{\circ}$ and HVA $<20^{\circ}$ and less than $50 \%$ subluxation of the medial sesamoid (grade 1).

b) Moderate HV was an IMA $>11$ but $<16^{\circ}$ and HVA of $20^{\circ}$ to $40^{\circ}$, with 50 to $75 \%$ subluxation of tibial sesamoid (grade 2).

c) Severe HV was an IMA $\geq 16^{\circ}$ and HVA of $>40^{\circ}$ and more than $75 \%$ subluxation of tibial sesamoid (grade 3 ). 
For each of these angles and tibial sesamoid positions, the difference $(\Delta)$ between preoperative and postoperative median values and the effectiveness of procedure correction (\%) was calculated.

\section{Statistical analysis}

Statistical analyses were performed by an independent statistician from the Department of Statistics at the University of Padua. The data is presented as the mean (plus standard deviation) or median (range) for continuous variables and as numbers for categorical measures. For the statistical evaluation of the angular values and the clinical scores obtained with the AOFAS scale pre-intervention and different follow-ups, we used the Student $t$ test. For angular values not normally distributed, we used the Wilcoxon test of signed ranks. The change in position of the medial sesamoid was analysed by testing the symmetry of Bowker, an extension of the McNemar test for tables larger than $2 \times 2$. All $p$ values were two-sided, using a significance level of $p<0.05$.

\section{Results}

Eighty feet, 43 right and 37 left, of 80 consecutively enrolled patients, met the inclusion criteria and were considered in the analyses. The median patient age at the time of the surgery was $51 \pm 15.5$ years (range 26-78). There were 75 women $(93.4 \%)$ and 5 men (6.6\%). None of the patients was lost during the different follow-ups.

\section{Clinical outcomes}

At the preoperative evaluation, the mean total AOFAS score of the patients treated was $54.1 \pm 8.3$ points (range 39-85). The median of the results was 52 points, and only 12 cases obtained over 60 points. Limitation in daily and recreational activities was implicated in 74 cases $(92.50 \%)$.

At the different follow-ups until the final one, the mean total AOFAS score of the patients treated improved progressively and significantly (Tables 1 and 2; Figs. 4 and 5): 72.20 points (range 44-100) at 3-month follow-up, 78.60 points (range 44-100) at 12-month follow-up and 87.15 points \pm 12.83 (range $52-100$ ) at the final follow-up $(p<0.0001)$. At the final follow-up, the pain was mild, occasional or absent in 73 cases (91.25\%). Only two patients $(2.50 \%)$ still had difficulty or limitation in daily and recreational activities. At the final follow-up period, the mean VAS score was $8.35 / 10(3-10)$.

\section{Radiographic outcomes}

According to the Mann and Coughlin grading system, 7 (8.75\%) patients were classified in group A (mild HV), 56 (70.0\%) in group B (moderate HV) and $17(21.25 \%)$ in group $\mathrm{C}$ (severe HV). The radiographic outcomes of our cohort are summarized in Table 3, while the radiographic results for each subgroup are reported in Table 4. Regarding bone/callus formation, complete healing of the osteotomies was noted in all series at 3-month follow-up (Fig. 6).

\section{Intermetatarsal angle (IMA)}

The mean IMA value decreased from $12.90^{\circ} \pm 2.83^{\circ}$ (range $7.50^{\circ}-20.00^{\circ}$ ) preoperatively to $9.00^{\circ} \pm 2.04^{\circ}$ (range $5^{\circ}-14^{\circ}$ ) at the 48-month follow-up (Fig. 7a), with a mean correction of $3.90^{\circ}$ and an effectiveness of $30.23 \%(p<0.05)$.

\section{Hallux valgus angle (HVA)}

The mean preoperative HVA was $26.40^{\circ} \pm 6.75^{\circ}$ (range $10^{\circ}-47.5^{\circ}$ ). The mean value at the 48 -month follow-up assessment was $13.90^{\circ} \pm 6.25^{\circ}$ (range $0^{\circ}-34.00^{\circ}$ ), with a mean correction of $12.50^{\circ}$ (Fig. $7 \mathrm{~b}$ ) and an effectiveness of $47.35 \%(p<0.05)$.

\section{Distal metatarsal articular angle (DMAA)}

The mean preoperative DMAA was $10.12^{\circ} \pm 4.26^{\circ}$ (range $3.5^{\circ}-26.00^{\circ}$ ). The mean value at the 48 -month follow-up examination was $5.40^{\circ} \pm 3.19^{\circ}$ (range $-1.00^{\circ}$ to $15.00^{\circ}$ ) with a mean correction of $4.72^{\circ}$ (Fig. 7c) and an effectiveness of $46.64 \%(p<0.05)$.

\section{Medial sesamoid position}

The mean preoperative dislocation of the medial sesamoid was $2.40 \pm 0.64$ (range $1-3$ ). Its mean value at the 48-month follow-up assessment was $1.30 \pm 0.63$ (range $0-3$ ), with a mean correction of 1.10 (range $0-3$ ) and an effectiveness of $45.83 \%$ ( $p<0.0001)$ (Fig. 7d).

\section{Articular surface congruency}

In the preoperative period, 61 patients $(76.25 \%)$ had a congruent articular surface and 19 (23.75\%) incongruent. At final follow-up, 77 patients $(96.25 \%)$ had a congruent articular surface and only 3 (3.75\%) incongruent.

\section{Metatarsal index}

In the preoperative period, the metatarsal index of patients was Minus (M1 < M2) in 34 cases $(42.5 \%)$, Plus Minus $(\mathrm{M} 1=\mathrm{M} 2)$ in 28 cases $(35.0 \%)$, and Major $(\mathrm{M} 1>\mathrm{M} 2)$ in 18 cases $(22.5 \%)$. At the last follow-up, it resulted Minus $(\mathrm{M} 1<\mathrm{M} 2)$ in 58 cases $(72.5 \%)$, Plus Minus

Table 1 AOFAS score at different follow-ups

\begin{tabular}{llcccc}
\hline & Preoperative & 3 months & 12 months & 48 months & $p$ value \\
\hline Mean AOFAS score $($ pts) & $54.1( \pm 8.3)$ & 72.2 & 78.6 & $87.1( \pm 12.8)$ & $p<0.0001$ \\
\hline
\end{tabular}


Table 2 AOFAS score before surgery and at final follow-up (48 months)

\begin{tabular}{|c|c|c|}
\hline & Preoperative (\%) & Last follow-up (\%) \\
\hline \multicolumn{3}{|l|}{ Pain } \\
\hline None & 2.5 & 62.5 \\
\hline Mild, occasional & 19 & 28.75 \\
\hline Moderate, daily & 78.5 & 8.75 \\
\hline Severe, almost always present & 0 & 0 \\
\hline \multicolumn{3}{|l|}{ Activity limitations } \\
\hline No limitations & 7.5 & 76.5 \\
\hline Limited daily and recreational activities & 60 & 21 \\
\hline Severe limitation & 32.5 & 2.5 \\
\hline \multicolumn{3}{|l|}{ Footwear requirements } \\
\hline Fashionable, conventional shoes & 13.5 & 44 \\
\hline Comfort footwear, shoe insert & 82.5 & 56 \\
\hline Modified shoes or brace & 4 & 0 \\
\hline \multicolumn{3}{|l|}{ MTP joint motion } \\
\hline Normal or mild restriction $>75^{\circ}$ & 82.5 & 61 \\
\hline Moderate restriction $30^{\circ}-74^{\circ}$ & 17.5 & 37.5 \\
\hline Severe restriction $<30^{\circ}$ & 0 & 1.5 \\
\hline \multicolumn{3}{|l|}{ Callus related to hallux MTP-IP } \\
\hline No callus or asymptomatic callus & 39 & 93.75 \\
\hline Callus symptomatic & 61 & 6.25 \\
\hline \multicolumn{3}{|l|}{ Alignment } \\
\hline Good, hallux well aligned & 0 & 68.75 \\
\hline Fair, no symptoms & 0 & 23.7 \\
\hline Poor obvious symptomatic malalignment & 100 & 7.5 \\
\hline
\end{tabular}

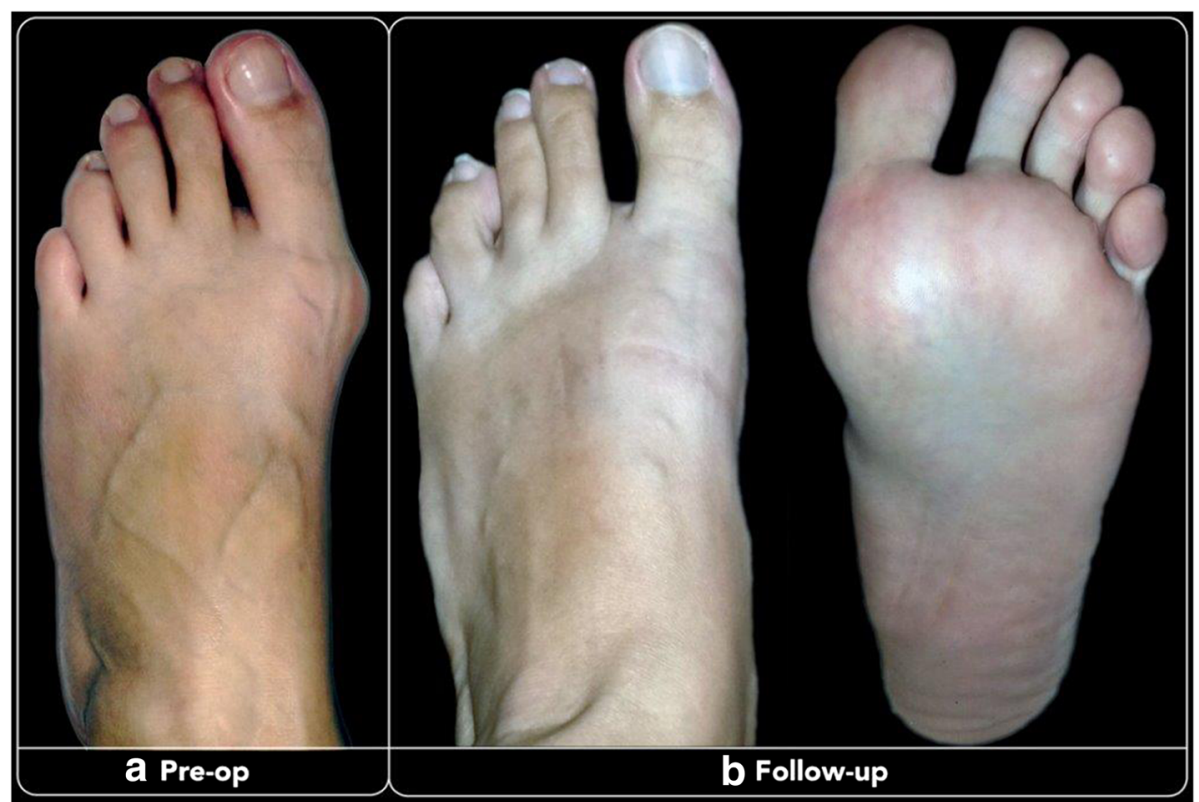

Fig. 4 Pre-op (a) and at 48-month last follow-up (b) clinical images of a 36-year-old woman after having undergone percutaneous Reverdin-lsham osteotomy, lateral release and Akin osteotomy for mild HV correction 


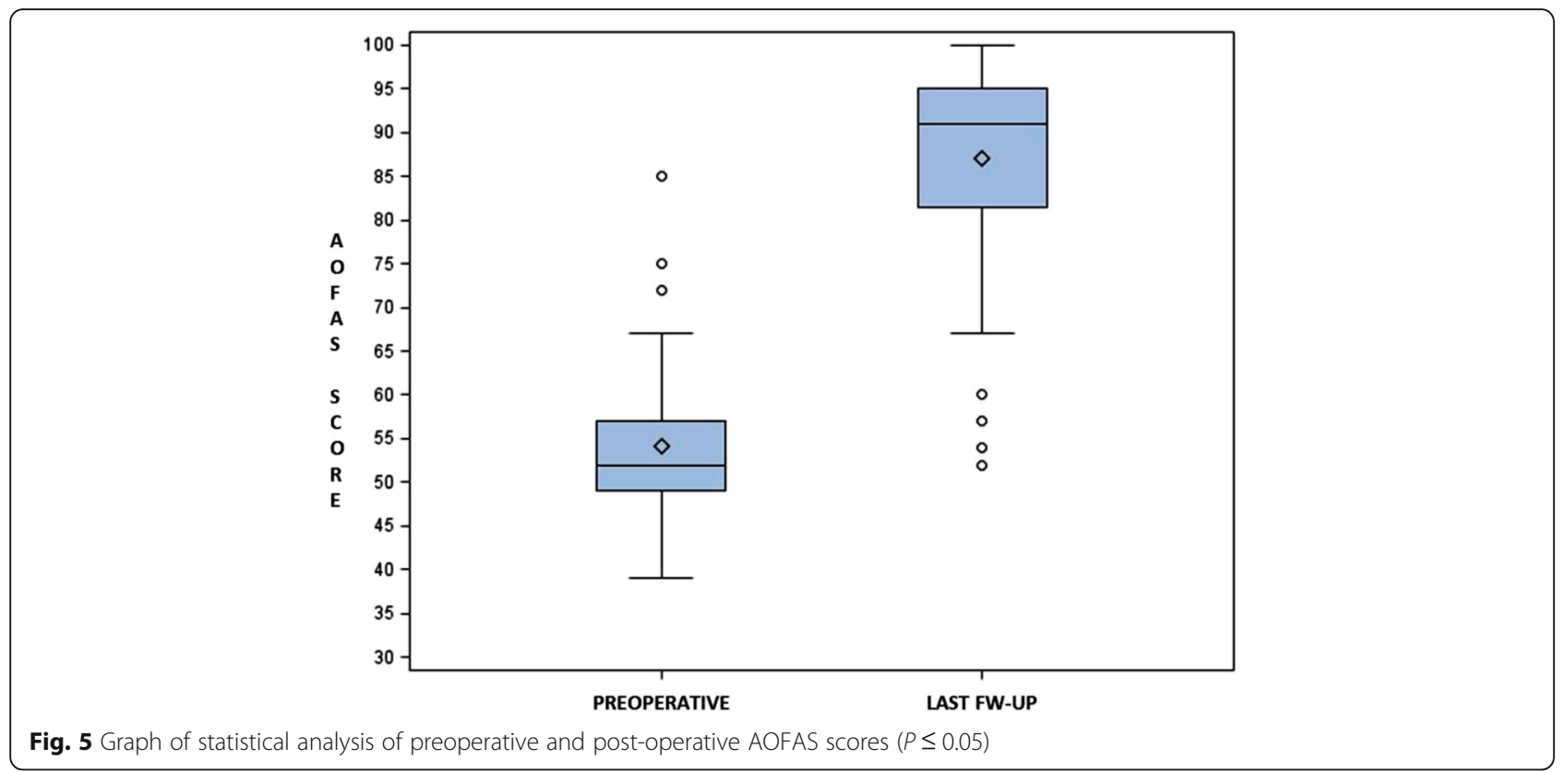

$(\mathrm{M} 1=\mathrm{M} 2)$ in 19 cases $(23.75 \%)$, and Major $(\mathrm{M} 1>\mathrm{M} 2)$ in 3 cases $(3.75 \%)$.

\section{Complications}

Complications occurred in 25 patients (31.25\%): six major and 19 minor. The major complications (7.5\%) included five cases of recurrence and one case of severe stiffness $\left(\mathrm{ROM}<30^{\circ}\right)$. The minor $(23.75 \%)$ complications were slight loss of normal range of MTP joint motion ( ROM $30^{\circ}-74^{\circ}$ ) in 16 cases. In three other cases, minor complications were resolved over time. Because of portal burns during operation, two patients presented delayed wound healing, which healed completely in 4 weeks and did not required subsequent surgery. One patient complained of dysesthesia of the skin distal to the interphalangeal joint of the big toe because of neuritis of a cutaneous sensory dorsal branch, an infrequent complication caused by incorrect surgical access, which had resolved spontaneously by the final follow-up. We did not encounter any cases of hallux varus due to overcorrection, malunion, delayed union or non-union. There were no cases of thrombo-embolism, no cutaneous or deep infections nor avascular necrosis of the metatarsal head. No case of dorsal displacement of the metatarsal head was recorded in this study.

\section{Discussion}

Although in the last few years, the number of studies regarding the effectiveness of MIS in HV correction has increased [35-37], to the best of our knowledge, this is the first prospective, single-centre study reporting outcomes of Reverdin-Isham percutaneous osteotomy in a consecutive, single surgeon, large patient series with a median follow-up of 48 months. Furthermore, no other report in the literature has assessed the results of this procedure at three different follow-ups. The study was designed to evaluate, on the basis of clinical and computer-assisted radiographic data, the validity and reliability of this percutaneous techniques for correction of mild to severe HV deformity.

In our cohort, the mean AOFAS score improved significantly until the last follow-up (Tables 1 and 2). An increase of 18.1 points 3 months after surgery was seen, further 6.7 points at 12 months and 33 points at the last follow-up. Moreover, more than half of the patients scored more than 90 points, while the median, statistically the more "powerful indicator," was 91 points, in contrast to 52 points in the preoperative period. This clinical improvement was also evident in all patient subgroups, almost in the same way, maintaining unaltered the gradient correlated to the degree of the deformity. In the sub-groups, the mean AOFAS score at the last follow-up was 87.1 points $(\Delta=+32.1)$ in the mild HV group, $86.6(\Delta=+32.7)$ in the moderate HV group and $83.7(\Delta=+30.2)$ in the severe one. Overall, the AOFAS score found in our study was comparable to those reported by different authors not only using minimally invasive techniques $[10,12,13,25,26,38]$, with or without osteosynthesis, but also with series of open surgical procedures using Chevron, Scarf or proximal metatarsal osteotomies [10, 13, 19, 39, 40]. In particular, our data was similar for patient demographic aspects and complications, including stiffness; however, our group of patients had a larger sample size and follow-up duration $[10,13,19,39]$. At the last follow-up, only seven patients reported daily pain, compared to 63 cases $(78.5 \%)$ of the 
Table 3 Angular values (IMA, HVA and DMAA), sesamoid position, metatarsal index and congruency of the metatarso-phalangeal-I articular surface

\begin{tabular}{|c|c|c|c|c|c|}
\hline & Pre-op & 3-month FU & 12-month FU & 48-month FU & Efficacy (\%) \\
\hline \multicolumn{6}{|l|}{ IMA (degrees) } \\
\hline Tot. (DS) & $12.9(2.8)$ & 9.0 & 9.0 & $9.0(2.0)$ & 30.23 \\
\hline Mild & 9.1 & & & 7.1 & 22.0 \\
\hline Moderate & 12.1 & & & 9.0 & 27.3 \\
\hline Severe & 16.6 & & & 10.0 & 39.2 \\
\hline \multicolumn{6}{|l|}{ HVA (degrees) } \\
\hline Tot. & $26.4(6.7)$ & 12.3 & 13.1 & $13.9(6.2)$ & 47.35 \\
\hline Mild & 16.4 & & & 9.8 & 40.2 \\
\hline Moderate & 26.0 & & & 14.2 & 45.0 \\
\hline Severe & 32.0 & & & 14.7 & 54.0 \\
\hline \multicolumn{6}{|l|}{ DMAA (degrees) } \\
\hline Tot. & $10.12(4.3)$ & 5.0 & 5.2 & $5.4(3.2)$ & 46.64 \\
\hline Mild & 6.3 & & & 3.9 & 38.1 \\
\hline Moderate & 10.0 & & & 5.3 & 47.0 \\
\hline Severe & 12.4 & & & 6.5 & 47.6 \\
\hline \multicolumn{6}{|c|}{ Sesamoid Position (pts) } \\
\hline Tot. & $2.4(0.6)$ & 1.1 & 1.2 & $1.3(0.6)$ & 45.83 \\
\hline Mild & 2.0 & & & 0.9 & 55.0 \\
\hline Moderate & 2.3 & & & 1.3 & 43.5 \\
\hline Severe & 2.8 & & & 1.7 & 39.3 \\
\hline \multicolumn{6}{|l|}{ Metatarsal Index } \\
\hline $\mathrm{M} 1<\mathrm{M} 2$ & 34 & & & 58 & \\
\hline$M 1=M 2$ & 28 & & & 19 & \\
\hline $\mathrm{M} 1>\mathrm{M} 2$ & 18 & & & 3 & \\
\hline \multicolumn{6}{|l|}{ MTP-I Art. Sup. } \\
\hline Congruent & 61 & & & 77 & \\
\hline Incongruous & 19 & & & 3 & \\
\hline
\end{tabular}

Table 4 Preoperative and last follow-up (48 months) angular values

\begin{tabular}{|c|c|c|c|c|}
\hline & Mild HV & Moderate HV & Severe HV & $p$ value \\
\hline \multicolumn{5}{|l|}{ IMA } \\
\hline Preoperative & $9.1( \pm 0.7)$ & $12.1( \pm 1.9)$ & $16.6( \pm 2.1)$ & $p<0.0001$ \\
\hline Last follow-up & $7.1( \pm 0.8)$ & $9.0( \pm 1.9)$ & $10.0( \pm 2.2)$ & $p<0.0001$ \\
\hline \multicolumn{5}{|l|}{ HVA } \\
\hline Preoperative & $16.4( \pm 3.1)$ & $26.0( \pm 4.93)$ & $32.0( \pm 7.7)$ & $p<0.0001$ \\
\hline Last follow-up & $9.8( \pm 5.8)$ & $14.2( \pm 5.9)$ & $14.7( \pm 7.3)$ & $p<0.0001$ \\
\hline \multicolumn{5}{|l|}{ DMAA } \\
\hline Preoperative & $6.3( \pm 1.22)$ & $10.0( \pm 4.0)$ & $12.4( \pm 4.7)$ & $p<0.0001$ \\
\hline Last follow-up & $3.9( \pm 2.5)$ & $5.3( \pm 3.0)$ & $6.5( \pm 3.9)$ & $p<0.0001$ \\
\hline \multicolumn{5}{|l|}{ Sesamoid position } \\
\hline Preoperative & $2.0( \pm 0.6)$ & $2.3( \pm 0.6)$ & $2.8( \pm 0.4)$ & $p<0.0001$ \\
\hline Last follow-up & $0.9( \pm 0.7)$ & $1.3( \pm 0.6)$ & $1.7( \pm 0.7)$ & $p<0.0001$ \\
\hline
\end{tabular}

preoperative period, while most of the subjects (73 cases; $91.5 \%)$ reported no pain or only mild occasional pain (Table 2). These percentages are similar to those of other studies [10, 24, 25]. Despite the anti-edema prophylaxis adopted, $40 \%$ of our subjects complained of swelling of the foot and ankle, which remained for more than a month after the operation. Apparently, the causes of both prolonged pain and swelling in these cases were related to the delayed formation of bone callus until the complete healing of the osteotomies seen at 3-month follow-up.

According to the AOFAS scale, the alignment was considered good in 55 cases (69\%), discrete in 19 cases $(23.5 \%)$ and poor in $6(7.5 \%)$. Overall, in $92.5 \%$ of our cases, there was improvement, which is comparable with that reported in the literature $[10,25,35,38-44]$ (Figs. 5 and 6).

One of the possible side effects of Reverdin-Isham percutaneous osteotomy, as it is an intra-articular medial 


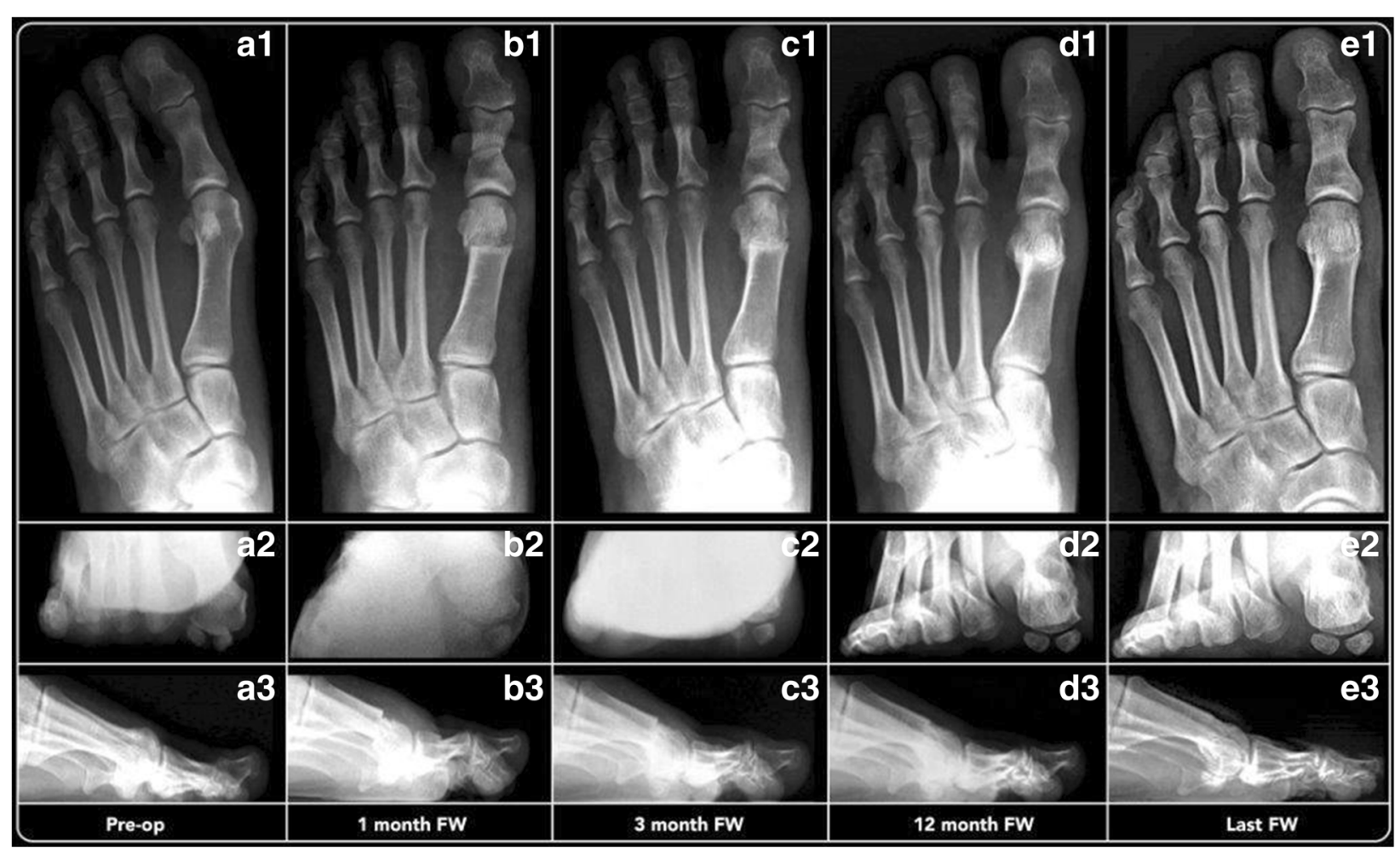

Fig. 6 A 36-year-old woman with mild HV: 1 antero-posterior radiographic images, 2 sesamoid and 3 lateral view at preoperative period (a), 1-month follow-up (b), 3-month follow-up (c), 12-month follow-up (d) and 48-month follow-up (e), showing the maintained correction of the deformity
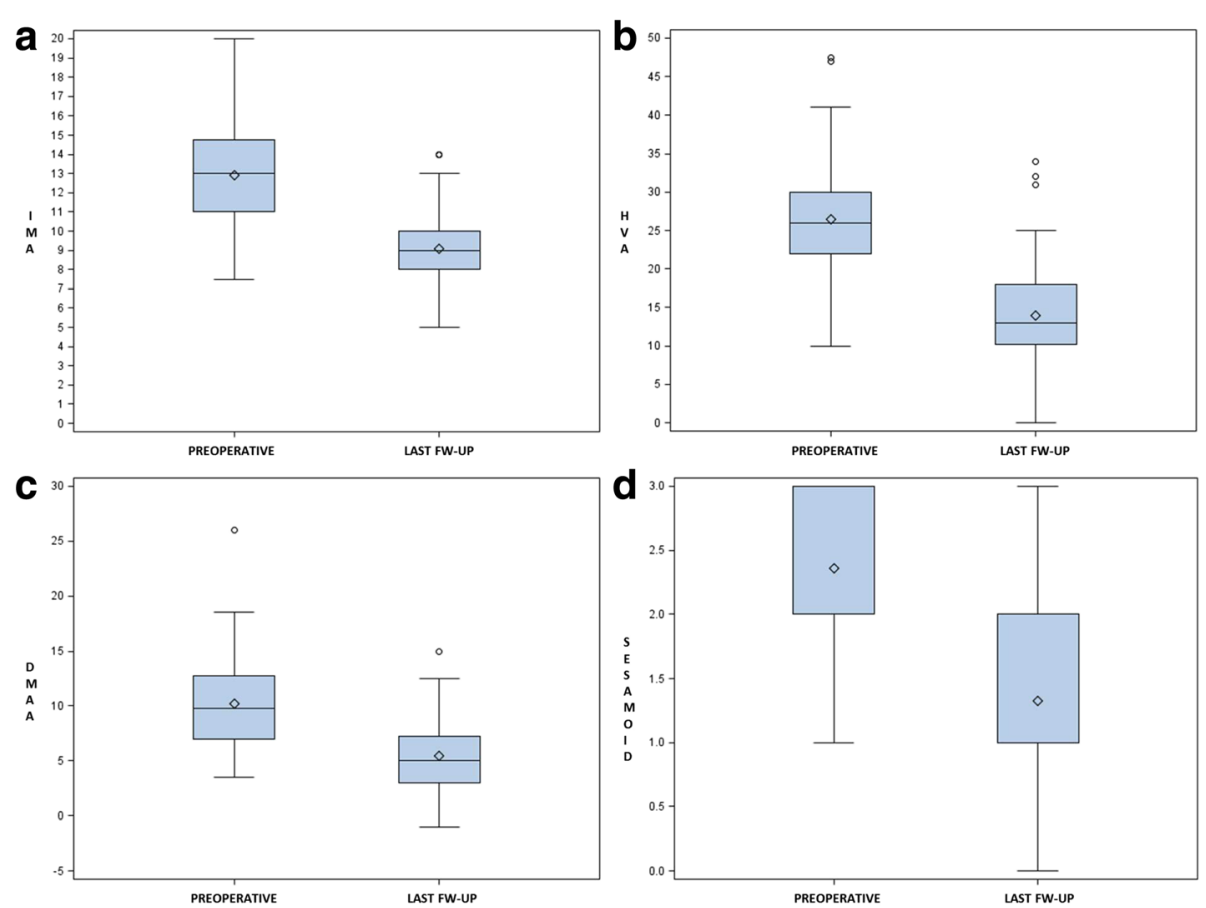

Fig. 7 Graph of statistical analysis of preoperative and post-operative different angular values ( $p \leq 0.05)$ : IMA (a), HVA (b), DMAA (c) and tibial sesamoid position $(\mathbf{d})$ 
closing wedge osteotomy, is the stiffness of the first metatarso-phalangeal joint $[45,46]$, which was noticed also in our cohort. Before the operation, 66 cases $(82.5 \%)$ presented with preserved range of motion and the other 14 cases (17.5\%) with slight limitation. After surgery, the joint movement was completely normal in 49 cases $(61 \%)$ and was slightly reduced in another 30 cases (37.5\%), while severe limitation to the flexion-extension was present only in an elderly patient. In accordance with Bauer et al., the potential cause could be the remains of bony fragments in the joint and the capsular tissues, produced during the extensive exostosectomy and the lack of accurate cleaning of the work area with rasps and irrigation with normal saline solution [35].

There were three intraoperative complications, which had resolved spontaneously by the final follow-up: one neuritis of a cutaneous sensory dorsal branch and two skin burns around the portal. Several studies have been reported in the literature $[47,48]$ comparing the complication rate between diathermy and scalpel for skin incision, without showing any significant differences. However, to the best of our knowledge, no study has examined the complication rate of surgical burr in MIS. In our experience, it is a fundamental surgical aspect to avoid putting too much manual pressure on the burr during the performance of osteotomies, rather accompanying it gently with the fingers during the entire process; otherwise, the resistant bone can push the burr to the edges of the portal, causing skin burn.

With regard to radiographic analysis (Table 3), all correction angular values obtained in our cohort were statistically significant $(p<0.0001)$. Although the distal closing wedge osteotomy has been described without resulting in any lateral translation of the metatarsal head, the IMA decreased from a mean value of $12.9^{\circ}$ at preoperative examination to a mean value of $9.0^{\circ}$ at the final follow-up, with a difference of $3.9^{\circ}$ and an efficacy of $30.5 \%$ in angular value correction. In agreement with Bauer et al. [10, 21, 35], the Reverdin-Isham osteotomy has a slight impact on this angle, reporting an IMA mean improvement of about just $3^{\circ}$, again a better mean correction of about $8^{\circ}$ and $15^{\circ}$ for the DMAA and HVA, respectively. According to our experience, the efficacy in IMA correction is probably explained by the combined action between the three different surgical steps of the procedure. First, during the application of manual force to perform the lateral cortex osteoclasis at the step of the wedge closing, a minimum translation of the metatarsal head occurs (Fig. 6 B1-E1). It is known that distal osteotomies allow achieving proximally $1^{\circ}$ of IMA correction for each millimetre of metatarsal head lateral translation $[49,50]$. Second, the tenotomy of the adductor hallucis tendon and lateral capsulotomy contributes to the lateral movement of the first metatarsal axis, further decreasing its varus. Third, Isham in his original paper of 1991, stated that the average reduction of the IMA is especially noted when the procedure is performed in association with Akin osteotomy [19].

For the HVA, the effectiveness in angular value correction was $47.4 \%$, with a preoperative mean value of $26.4^{\circ}$ and $13.9^{\circ}$ at the last follow-up, respectively. One of the objectives of distal osteotomies is to reduce the DMAA by a medial rotation of the metatarsal head. In the analysed sample, average correction efficacy was $46.1 \%$; the mean preoperative angular value was $10.2^{\circ}$ and $5.4^{\circ}$ at the final follow-up. According to Coughlin [29], it is very important to correct DMAA, since a stable recovery can be achieved only by setting up the bone structure, reorienting articular surfaces and re-equilibrating muscle forces of the first ray, avoiding the retraction of soft tissue and peri-articular adhesions. As reported in the literature $[10,24,35,39,42]$, confirmation of the re-orientation of the forces on the muscle-ligamentous compartment was seen as restoration of the articular congruency and sesamoid compartment alignment.

Reverdin-Isham percutaneous osteotomy was a reliable procedure in correcting the different radiological parameters considered in this analysis, and our data are comparable with the reported angular corrections obtained with other percutaneous or open distal metatarsal osteotomies [13, 39, 42, 51]. Derotation of the metatarsal head (DMAA), and the anatomical reduction of the tibial sesamoid, necessary to prevent the recurrence of valgus [52], were all maintained until the last follow-up (Table 3). However, the results obtained in the correction of the HV severe deformities were less encouraging. In fact, the correction efficacy of the different single angles analysed, although high, was in some cases not sufficient to report them as in the normal range (Table 4). The six major complications of our series occurred in the severe forms, with an IMA greater than $15^{\circ}$. Hence, in accordance with Bauer et al. [10], we believe this is the angular value limit, beyond which the use of only Reverdin-Isham osteotomy as described is not recommended.

The first strength of this study is its nature: a prospective evaluation of a consistent group of 80 patients with the same fixed follow-up points. A 48-month follow-up can be considered a long follow-up period compared with previous published studies. Further, all operations and the post-operative controls were always performed in the same way by the same surgeon (C.B.). All clinical and radiographic data were collected and analysed separately by the same two independent investigators, who were not involved in the patients' treatment and one not belonging to our unit. The main limitation of this study is the lack of a control group, which would be useful to compare the results of this percutaneous technique. However, as reported by several authors $[15,42,44,53]$, MIS includes different 
techniques, and the heterogeneity of the groups examined in various reports does not permit at present an effective comparison and clear conclusions. For these reasons, we believe that long-term follow-up with multicentre studies and randomized controlled clinical trials comparing Reverdin-Isham osteotomy outcomes to those of other percutaneous methods would provide useful information for the validity and reliability of MIS in the treatment of forefoot deformities.

\section{Conclusions}

Based on our experience in MIS with the first cohort of patients described in this report, we conclude that Reverdin-Isham and Akin percutaneous osteotomy in combination with previous exostosectomy and following lateral soft-tissue release is a safe, effective and reliable procedure for correction of mild-to-moderate symptomatic HV. Good results remained consistent at the midterm follow-up point. The most important aspects that should encourage the use of this percutaneous technique are its minimally invasive nature, the low number of complications, the absence of osteosynthesis, distal ankle block anaesthetic technique, early weight bearing and good cosmetic results with minimal surgical scars.

\section{Abbreviation}

AOFAS: American Orthopaedic Foot and Ankle Society; DMAA: Distal Metatarsal Articular Angle; GRECMIP: Groupe de Recherche et d'Enseignement en Chirurgie Mini-Invasive du Pied; HV: Hallux Valgus; HVA: Hallux Valgus Angle; IMA: Intermetatarsal Angle; MIS: Minimally Invasive Surgery; VAS: Visual Analogue Scale

\section{Acknowledgements}

The authors acknowledge Prof. Anna Chiara Frigo for her assistance with the statistical analysis and Mr Renzo Banzato for his assistant in taking pictures.

\section{Funding}

None.

\section{Availability of data and materials}

The dataset supporting the conclusions of this article is available at our institution.

\section{Authors' contributions}

CB contributed to the study concept and design and wrote the paper. MF and MGR carried out the data collection and analysis. MDP participated in the figure design and review. MC interpreted the data. RA and PR participated in the final review. The manuscript has been read and approved by all of the listed authors, who declare that this article represents honest work.

\section{Competing interests}

The authors declare that they have no competing interests.

\section{Consent for publication}

The patients gave their oral and written informed consent to the publication of their anonymous and clustered data and anonymous pictures.

\section{Ethics approval and consent to participate}

This clinical practice observational study was performed in accordance with the ethical standards of the 1964 Declaration of Helsinki as revised in 2000 and those of Good Clinical Practice. Further, the approval from the Padua Hospital General Clinical Directorate was obtained to introduce the novel technique before starting the operations and analyse their clinical and radiographic outcomes during the study. For these reasons, ethics approval of
Padua Hospital Ethical Committee was not mandatory. All patients received a thorough explanation of this study and informed consent to participate was obtained from the participants.

\section{Author details}

'Orthopaedic Clinic, Department of Surgery, Oncology and Gastroenterology DiSCOG, University of Padua, via Giustiniani 2, 35128 Padova, Italy. ${ }^{2}$ Human Anatomy and Embryology Unit, Experimental Pathology and Therapeutics Department, University of Barcelona, Barcelona, Spain. ${ }^{3}$ Health Sciences Faculty of Manresa, University of Vic-Central University of Catalunya, Barcelona, Spain. ${ }^{4}$ Orthopaedic and Trauma Unit, Padua Hospital, via Giustiniani 2, Padova, Italy.

Received: 6 September 2016 Accepted: 18 November 2016

Published online: 05 December 2016

\section{References}

1. Mann RA, Coughlin MJ. Hallux valgus-etiology, anatomy, treatment and surgical considerations. Clin Orthop Relat Res. 1981;157:31-41.

2. Root M, Orien W, Weed J. Normal and abnormal function of the foot, vol. 2. Los Angeles: Clinical Biomechanics Corp; 1977.

3. Biz C, Favero L, Stecco C, Aldegheri R. Hypermobility of the first ray in ballet dancer. Mus Lig Tend J. 2012;2(4):282-8.

4. Baravarian B, Brinski GB, Burns P. Lapidus bunionectomy: arthrodesis of the first metatarsocuneiform joint. Clin Podiatr Med Surg. 2004;21:97-111.

5. Richardson EG. Foot in adolescent and adults. In: Crenshaw AH, editor. Campbell's operative orthopedics, vol. 2. 7th ed. St Louis: C.V. Mosby; 1992. p. 829-988.

6. Stienstra JJ. Large displacement distal chevron osteotomy, for the correction of hallux valgus deformity. J Foot Ankle Surg. 2002:41:213-20.

7. Biz C, Corradin M, Petretta I, Aldegheri R. Endolog technique for correction of hallux valgus: a prospective study of 30 patients with 4-years follow-up. J Orthop Surg Res. 2015;10:102-15.

8. Trnka HJ. Osteotomies for hallux valgus correction. Foot Ankle Clinics N Am. 2005:10:15-33.

9. Roukis TS. Percutaneous and minimum incision metatarsal osteotomies: a systematic review. J Foot Ankle Surg. 2009:48(3):380-7.

10. Bauer $T$, de Lavigne $C$, Biau D, De Prado M, Isham S, Laffenetre $O$. Percutaneous hallux valgus surgery: a multicenter study of a 189 cases. Orthop Clin N Am. 2009;40:505-14.

11. Dennis NZ, Das De S. Modified Mitchell's osteotomy for moderate to severe hallux valgus-an outcome study. J Foot Ankle Surg. 2011;50:50-4.

12. Giannini S, Faldini C, Vannini F, Digennaro V, Bevoni R, Luciani D. The minimally invasive osteotomy "S.E.R.I." (simple, effective, rapid, inexpensive) for correction of bunionette deformity. Foot Ankle Int. 2008;29(3):282-6.

13. Magnan B, Bortolazzi R, Samaila E, Pezzè L, Rossi N, Bartolozzi P. Percutaneous distal metatarsal osteotomy for correction of hallux valgus. Surgical technique. J Bone Joint Surg Am. 2006:88-A(suppl-1):135-48.

14. Siclari A, Decantis V. Arthroscopic lateral release and percutaneous distal osteotomy for hallux valgus: a preliminary report. Foot Ankle Int. 2009;30:675-9.

15. Maffulli N, Longo UG, Marinozzi A, Denaro V. Hallux valgus: effectiveness and safety of minimally invasive surgery. A systematic review. Br Med Bull. 2011;9:149-67.

16. De Prado M, Ripoll PL, Golano P. Minimally invasive foot surgery: surgical techniques, indications, anatomical basis. About your health, Spain. 2009. p. 63-123.

17. Van Enoo RE, Cane EM. Minimal incision surgery: a plastic technique or a cover-up? Clin Podiatr Med Surg. 1986:3(2):321-35.

18. Hymes L. Introduction: brief history of the use of minimum incision surgery (MIS). In forefoot minimum incision in podiatric medicine: a handbook on primary corrective procedures on the human foot using minimum incisions with minimum trauma. New York: Futura Publishing Co.; 1977.

19. Isham SA. The Reverdin-Isham procedure for the correction of hallux abducto valgus. A distal metatarsal osteotomy procedure. Clin Podiatr Med Surg. 1991;8-1:81-94.

20. de Prado M, Ripoll PL, Golanó P. Minimally invasive surgery. Barcelona: AYH Publisher; 2009. p. 3-123.

21. Bauer T. Percutaneous forefoot surgery. Orthop Traumatol Surg Res. 2014;100(1 Suppl):S191-204. 
22. Laffenetre $\mathrm{O}$, et al. Chirurgie par technique percutanée de l'avant-pied: hallux valgus, metatarsalgies, 5e rayon. In: Lemrijse $T$, Valtin $B$, editors. Pathologie du pie et de la cheville. Paris: Masson; 2009. p. 243-53.

23. Magnan B, Samaila E, Merlini M, Bondi M, Mezzari S, Bartolozzi P. Percutaneous distal osteotomy of the fifth metatarsal for correction of bunionette. J Bone Joint Surg Am. 2011;93:2116-22

24. De Prado M, Ripoll PL, Vaquero J, Golàno P. Tratamiento quirùrgico percutàneo del hallux valgus mediante osteotomias mùltiples. Rev Esp Cir Ortop Traumatol. 2003;47:406-16.

25. Lucas y Hernandez J, Golanó P, Roshan-Zamir S, Darcel V, Chauveaux D, Laffenêtre $\mathrm{O}$. Treatment of moderate hallux valgus by percutaneous, extraarticular reverse-L Chevron (PERC) osteotomy. Bone Joint J. 2016;98-B:365-73.

26. Laffenetre $O$, et al. 1st GRECMIP. Mini-invasive surgery of hallux valgus. In: Valtin B, Leemrijse T, editors. Chirurgie de l'avant-pied. 2nd ed. Paris: Elsevier; 2005. p. 96-104.

27. Smith RW, Reynolds JC, Stewart MJ. Hallux valgus assessment: report of research committee of American Orthopaedic Foot and Ankle Society. Foot Ankle. 1984;5(2):92-103.

28. Kitaoka HB, Alexander IJ, Adelaar RS, Nunley JR, Myerson MS, Sanders M. Clinical rating systems for the ankle-hindfoot, midfoot, hallux, and lesser toes. Foot Ankle Int. 1994;15:349-53.

29. Coughlin MJ, Mann RA, Saltzman CL. Surgery of the foot and ankle. 8th ed. Philadelphia: Mosby Elsevier; 2007. p. 184-19.

30. Vernois J, Redfern D. Percutaneous Chevron: the union of classic stable fixed approach and percutaneous technique. Fuss Sprunggelenk. 2013;11:70-5.

31. Sullivan BT, Robison JB, Palladino SJ. Interevaluator variability in the measurement of proximal articular set angle. J Foot Surg. 1988;2:466-8.

32. Shechter DZ, Doll PJ. Tangential angle to the second axis. A new angle with implications for bunion surgery. J Am Podiatr Med Assoc. 1985;75:505-12.

33. Bonnel F, Canovas F, Poierre G. Radiographical results of scarf osteotomy for hallux valgus related to distal metatarsal articular angle. Rev ChirOrthop. 1999:85:381-6

34. Nery C, Réssio C, de Azevedo Santa Cruz G, de Oliveira RS, Chertman C. Proximal opening-wedge osteotomy of the first metatarsal for moderate and severe hallux valgus using low profile plates. Foot Ankle Surg. 2013:19(4):276-82.

35. Bauer T, Biau D, Lortat-Jacob A, Hardy P. Percutaneous hallux valgus correction using the Reverdin-Isham osteotomy. Orthop Traumatol Surg Res. 2010;96:407-16.

36. de Lavigne C, Guillo S, Laffenetre 0 , et al. The treatment of hallux valgus with the mini-invasive technique. Interact Surg. 2007;2:31-7.

37. Vernois J. The treatment of the hallux valgus with a percutaneous chevron osteotomy. J Bone Joint Surg (Br). 2011;93-B(supp-IV):482.

38. Faour-Martín O, Martín-Ferrero MA, Valverde García JA, Vega-Castrillo A, de la Red-Gallego MA. Long-term results of the retrocapital metatarsal percutaneous osteotomy for hallux valgus. Int Orthop. 2013;37:1799-803.

39. Magnan B, Pezzè L, Rossi N, Bartolozzi P. Percutaneous distal metatarsal osteotomy for correction of hallux valgus. J Bone Joint Surg. 2005;87:1191-9.

40. Deenik AR, Pilot P, Brandt SE, van Mameren H, Geesink RG, Draijer WF. Scarf versus chevron osteotomy in hallux valgus: a randomized controlled trial in 96 patients. Foot Ankle Int. 2007;28(5):537-41.

41. Gonzalez Lopez JJ, Rodriguez Rodriguez S. Functional, esthetic and radiographic results of treatment of hallux valgus with minimally invasive surgery. Acta Ortop Mex. 2005;19(1):S42-6.

42. Maffulli N, Oliva F, Coppola C, Miller D. Minimally invasive hallux valgus correction: a technical note and a feasibility study. J Surg Orthop Adv. 2005; 14:193-8.

43. Kerr HL, Jackson R, Kothari P. Scarf-Akin osteotomy correction for hallux valgus: short-term results from a district general hospital. Foot Ankle Surg. 2010;49:16-9.

44. Maffulli N, Longo UG, Oliva F, Denaro V, Coppola C. Boschosteotomy and scarf osteotomy for hallux valgus correction. Orthop Clin North Am. 2009;40:515-24.

45. Meier PJ, Kenzora JE. The risks and benefits of distal first metatarsal osteotomies. Foot Ankle. 1985;6:7-17.

46. Laughlin TJ. Complications of distal first metatarsal osteotomies. Foot Ankle Surg. 1996;34:524-31.

47. Shamim M. Diathermy vs. scalpel skin incisions in general surgery: doubleblind, randomized, clinical trial. World J Surg. 2009;33(8):1594-9.

48. Ly J, Mittal A, Windsor J. Systematic review and meta-analysis of cutting diathermy versus scalpel for skin incision. Br J Surg. 2012;99(5):613-20.
49. Sarrafian SK. A method of predicting the degree of functional correction of the metatarsus primus varus with a distal lateral displacement osteotomy in hallux valgus. Foot Ankle. 1985;5:322-6.

50. Badwey TM, Dutkowsky JP, Graves SC, Richardson EG. An anatomical basis for the degree of displacement of the distal chevron osteotomy in the treatment of hallux valgus. Foot Ankle Int. 1997;18(4):213-5.

51. Jones CP, Coughlin MJ, Grebing BR, Kennedy MP, Shurnas PS, Viladot R, Golanó P. First metatarsophalangeal joint motion after hallux valgus correction: a cadaver study. Foot Ankle Int. 2005;26:614-9.

52. Okuda R, Kinoshita M. Postoperative incomplete reduction of the sesamoids as a risk factor for recurrence of hallux valgus. J Bone Joint Surg Am. 2009;91:1637-45

53. Oliva F, Longo UG, Maffulli N. Minimally invasive hallux valgus correction. Orthop Clin North Am. 2009;40(4):525-30. doi:10.1016/j.ocl.2009.06.005. x.

\section{Submit your next manuscript to BioMed Central and we will help you at every step:}

- We accept pre-submission inquiries

- Our selector tool helps you to find the most relevant journal

- We provide round the clock customer support

- Convenient online submission

- Thorough peer review

- Inclusion in PubMed and all major indexing services

- Maximum visibility for your research

Submit your manuscript at www.biomedcentral.com/submit
Biomed Central 